\title{
25 Research Square \\ Fourth thoracic vertebra as landmark for depth of right internal jugular vein catheterization in infants
}

\section{Guoliang Liu}

Beijing Children's Hospital

Jianmin Zhang

Beijing Children's Hospital

Fang Wang ( $\sim$ bjetyymzk@163.com )

Beijing Children's Hospital

Heqi Liu

Beijing Children's Hospital

\section{Research Article}

Keywords: catheterization, central venous catheter, infant, jugular vein, radiography, vertebra

Posted Date: March 8th, 2022

DOI: https://doi.org/10.21203/rs.3.rs-1233599/v1

License: (9) This work is licensed under a Creative Commons Attribution 4.0 International License.

Read Full License 


\section{Abstract}

Background $\mathbb{X}$ The carina is considered a reliable marker for the depth of right internal jugular vein catheterization in infants on chest radiograph. In adult anatomy, the carina is typically located at the level of the fifth thoracic vertebra. We are not aware of a positional relationship between infant carina and thoracic vertebrae. Thus, we evaluated that a vertebral body may be at the same level as carina and can be as radiographic landmarks for the depth of right internal jugular vein catheterization in infants.

Methods $₫$ In this retrospective analysis, 108 infants (aged 1-12 months) who underwent congenital heart surgery between January 1, 2019 and June 30, 2019 were included. We analyzed the post-operative chest radiographs of those who underwent right internal jugular vein catheterization and assessed the positional relationship of the carina and vertebral bodies.

Results $₫$ In total, 95 children were enrolled; The carina was located at the third thoracic vertebra in 2 cases (2\%) and at the fourth thoracic vertebra in 93 cases (98\%). There was a significant difference in the position of the carina $(\mathrm{P}=0.01, P<0.05$; respectively).

Conclusions $₫$ Most fourth thoracic vertebrae were at the same level as the carina on chest radiographs. Therefore, it has potential as a radiographic landmark for the depth of right internal jugular vein catheterization in infants on chest radiograph.

\section{Introduction}

Central venous catheterization is an important cardiac surgery technique. The right internal jugular vein is the most used central venous catheter (CVC) placement site in infants; due to their relatively small size and short superior vena cava (SVC) length, determining catheterization depth requires a high level of precision to avoid arrhythmia, heart injury, and surgical interference. ${ }^{1,2}$ When such catheterization is too shallow, it leads to inaccurate measurement of CVP and poor fluid and blood transfusion; the catheter may even be pulled out. An anatomical measurement of infant cadavers revealed that the carina is located $22 \mathrm{~mm}$ above the junction of the SVC and right atrium, ${ }^{3}$ while clinical studies have revealed that the optimal depth of CVC tip placement is at the level of the carina on chest radiographs. ${ }^{3-8}$ However, not all carina are particularly clear on chest radiographs, either for radiologic reasons or for patient reasons, and the position of carina varies with aspiration and affects the depth of puncture localization. In adult anatomy, the carina is typically located at the level of the fifth thoracic vertebra. ${ }^{9}$ We are not aware of a positional relationship between infant carina and thoracic vertebrae. We hypothesized that a vertebral body may be at the same level as carina and serve as a radiographic landmark for the depth of right internal jugular vein catheterization on a chest radiograph.

\section{Methods}


This study was approved by the Ethics Committee of the Beijing Children's Hospital, China (No. 2020-K015). Informed patient consent was waived by the Ethics Committee of the Beijing Children's Hospital. All the experiment protocol for involving human date and methods were in accordance with the guidelines of Declaration of Helsinki. We retrospectively analyzed 108 children aged between 1 and 12 months who underwent cardiac surgery and routinely took radiograph post-operation from January 1, 2019 to June 30, 2019. Informed patient consent was waived due to the retrospective nature of the study.

In this retrospective study, we aimed to analyze the relationship between the carina and thoracic vertebra in infants to determine the presence of a vertebral body, at the same level as the carina, that could be used as a radiographic landmark to guide the depth of right internal jugular vein catheterization in infants. In total, 98 cases of right internal jugular vein catheterization were confirmed via postoperative chest radiography. The first post-operation chest radiograph of each child was analyzed using the Carestream Picture Archive and Communication System (PACS; Carestream Health Inc., Rochester, NY).

All measurements were performed by one experienced radiologist using the hospital's PACS software; all catheters were inserted under general anesthesia. Catheterization was performed by anesthesiologists, experienced in right internal jugular vein catheterization. Due to the retrospective nature of the study, details regarding catheter placement were not clear.

\section{Statistical analysis}

Results are expressed as number of patients, percentage, mean \pm standard deviation, and median (interquartile range-IQR) where appropriate. Percentage of carina located at thoracic vertebrae were compared using chi-squared test. Statistical analysis was performed with SPSS Statistics for Mac version 21 (IBM, Armonk, NY), and $\mathrm{P}<0.05$ was considered statistically significant.

\section{Results}

We reviewed the radiographs of 108 consecutive infants who underwent cardiac surgery; chest radiographs revealed that 98 of these patients underwent right internal jugular vein catheterization. Due to the overlap of the CVC and other cardiac leads, the data of three infants could not be assessed; therefore, we enrolled and assessed 95 infants, as indicated in Fig. 1. The infants' sex, age, weight, and medical diagnosis are summarized in Table 1. 
Table 1

Infant demographic characteristics

\begin{tabular}{|ll|}
\hline Variable & Value \\
\hline Age, median (IQR), months & $4(3-5)$ \\
\hline Weight, mean \pm standard deviation, kg & $5.6 \pm 1.4$ \\
\hline Sex, male/female, No. (\%) & $56 / 39(59 / 41)$ \\
Diagnosis, No. (\%) & $66(70)$ \\
Ventricular septal defect & $2(2)$ \\
Atrial septal defect & $4(4)$ \\
Tetralogy of Fallot & $4(4)$ \\
Total anomalous pulmonary venous connection & $5(5)$ \\
Patent ductus arteriosus & $5(5)$ \\
Aortic coarctation & $9(10)$ \\
Others & \\
\hline Abbreviations: IQR, interquartile range & \\
\hline
\end{tabular}

Analysis of the 95 cases the carina was located at the third thoracic vertebra in 2 case (2\%), and the fourth thoracic vertebra in 93 cases (98\%), as indicated in Fig. 2; this difference in carina location was statistically significant $(P=0.01, P<0.05$, respectively; Fig. 3$)$.

\section{Discussion}

In this study, we retrospectively analyzed the postoperative chest radiographs of infants who underwent cardiac surgery and assessed the possibility of using a vertebral body as a radiographic landmark for the identification of the depth of right internal jugular vein catheterization. We found that the fourth thoracic vertebra is at the same level as the carina in most infants, and it can be a potential useful radiographic indicator of the depth of catheterization of the right internal jugular vein in infants on chest radiograph.

CVC insertion, including internal jugular vein catheterization, femoral vein catheterization, and subclavian catheterization, is a very important perioperative technique. Currently, the most widely utilized form is right internal jugular vein catheterisation. ${ }^{10}$ The optimal timing of surgical treatment for children with certain congenital heart diseases is during infancy. Infants have a relatively large head and short neck; therefore, it is difficult to insert and place the right internal jugular vein catheter, especially in terms of the appropriate depth. There have been reports that improper CVC placement can cause serious complications in infants. ${ }^{11-13}$ 
According to current literature, the carina can be used as a reliable reference index for the depth of right internal jugular vein puncture on chest radiograph. However, we all know bone tissue is more recognizable than cartilage tissue under $x$-rays, so vertebral bodies are more visible clearly than the carina on chest radiographs. And the position of carina changes with the infants ' breathing, but the position of the vertebral body does not .And in this study, we discovered that the fourth thoracic vertebra was at the same level as the carina in most cases $(98 \%)$, contrary to what is observed in adults ${ }^{9}$; this discrepancy may be due to differences in the physical development stages of infants and adults. Our results suggest that the fourth thoracic vertebra can also be used as a radiographic marker for optimal CVC positioning, which may reduce complications.

There are certain limitations in this study. This is a retrospective study, some important information about the patient was not collected completely, resulting in some singleness of data in our analysis. Ninety-five children were a small sample size for a retrospective study, but a significant number for infants. Of course, we would like to consolidate our findings by including more infants in the future if possible. Finally, we only focused on infants; our results are therefore not necessarily applicable to children of all ages. These limitations highlight the need and scope for further, preferably prospective, studies on this topic.

\section{Conclusion}

In conclusion, the fourth thoracic vertebra can be as a radiographic landmark for the depth of catheterization in the right internal jugular vein of infants on chest radiography. This feature may be valuable for reducing the clinical risks associated with inappropriate CVC placement.

\section{Declarations}

Funding. This research received no specific grant from any funding agency in the public, commercial or not-for-profit sectors.

Competing interests: The authors declare that they have no competing interests.

Authors' contributions: FW contributed to the overall study design, data analysis, and manuscript revision. GLL helped design the study, performed the experiments, collected and analysed data, and wrote the manuscript. JMZ helped design the study and revised the manuscript. HQL performed the experiments and collected data. All authors read and approved the final manuscript.

Ethics approval and consent to participate: This study was approved by the Ethics Committee of the Beijing Children's Hospital, China (No. 2020-K-015). Informed patient consent was waived by the Ethics Committee of the Beijing Children's Hospital.

Data Availability Statements. The datasets generated during and analysed during the current study are available from the corresponding author on reasonable request. 


\section{References}

1. Vesely TM. Central venous catheter tip position: a continuing controversy. J Vasc Interv Radiol. 2003;14:527-34. doi: 10.1097/01.rvi.0000071097.76348.72.

2. Nowlen TT, Rosenthal GL, Johnson GL, Tom DJ, Vargo TA. Pericardial effusion and tamponade in infants with central catheters. Pediatrics. 2002;110:137-42. doi: 10.1542/peds.110.1.137.

3. Albrecht K, Breitmeier D, Panning B, Tröger HD, Nave H. The carina as a landmark for central venous catheter placement in small children. Eur J Pediatr. 2006;165:264-266. doi: 10.1007/s00431-0050044-5.

4. Xu H, Zhu X, Li Jet al. Using sternal angle as anatomic landmark for right internal jugular vein catheterisation in pediatrics. Acta Anaesthesiol Scand. 2019;00:1-5. doi: 10.1111/aas.13474.

5. Schuster M, Nave H, Piepenbrock S, Pabst R, Panning B. The carina as a landmark in central venous catheter placement. Br J Anaesth. 2001(2):192-194. doi: 10.1093/bja/85.2.192.

6. Yoon SZ, Shin JH, Hahn S, et al. Usefulness of the carina as a radiographic landmark for central venous catheter placement in paediatric patients. Br J Anaesth. 2005;95:514-517. doi: 10.1093/bja/aei199.

7. Stonelake, $P$ A, Bodenham AR. The carina as a radiological landmark for central venous catheter tip position. Br J Anaesth. 2006;96(3):335-340. doi: 10.1093/bja/aei310.

8. Murali B H, Srividya G S, Reshmavathi V. Appropriate length of the central venous catheter insertion via right internal jugular vein in children using external anatomical. Curr Pediatric Res. 2016;20:2023.

9. Bryce Mieczkowski, Brian F. Seavey. StatPearls [Internet]. StatPearls Publishing; Treasure Island (FL):Feb. 5, 2020. Anatomy, Head and Neck, Trachea. Accessed May 23, 2021.

10. Karaaslan P, Darcın K, Ormecı T, et al. Effects of varying entry points and trendelenburg positioning degrees in internal jugular vein area measurements of newborns. Niger J Clin Pract. 2018;21(4):514518.

11. Askegard-Giesmann JR, Caniano DA, Kenney BD. Rare but serious complications of central line insertion. Semin Pediatr Surg. 2009;18:73-83. doi: 10.1053/j.sempedsurg.2009.02.003.

12. Hodzic S, Golic D, Smajic J, Sijercic S, Umihanic S, Umihanic S. Complications related to insertion and use of central venous catheters (CVC). Med Arch. 2014;68:300-303. doi:

10.5455/medarh.2014.68.300-303.

13. Nayeemuddin $M$, Pherwani AD, Asquith JR. Imaging and management of complications of central venous catheters. Clin Radiol.2013;68:529-544. doi: 10.1016/j.crad.2012.10.013.

\section{Figures}




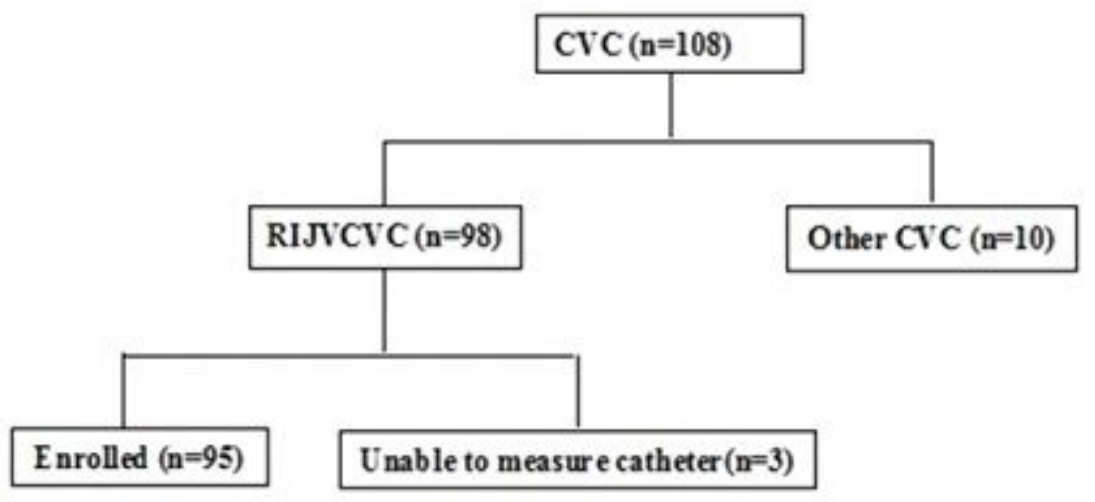

Figure 1

Details of assessed CVC cases

CVC, central venous catheter; RIJV, right internal jugular vein

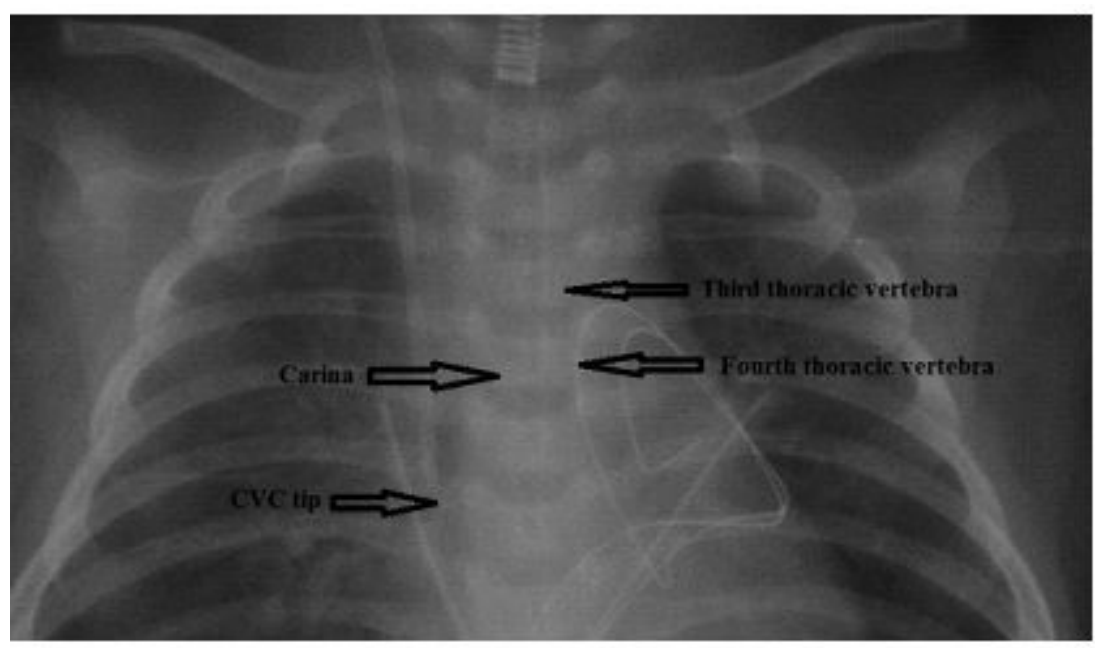

Figure 2

Carina, CVC tip and thoracic vertebra display on the radiograph .

CVC, central venous catheter 


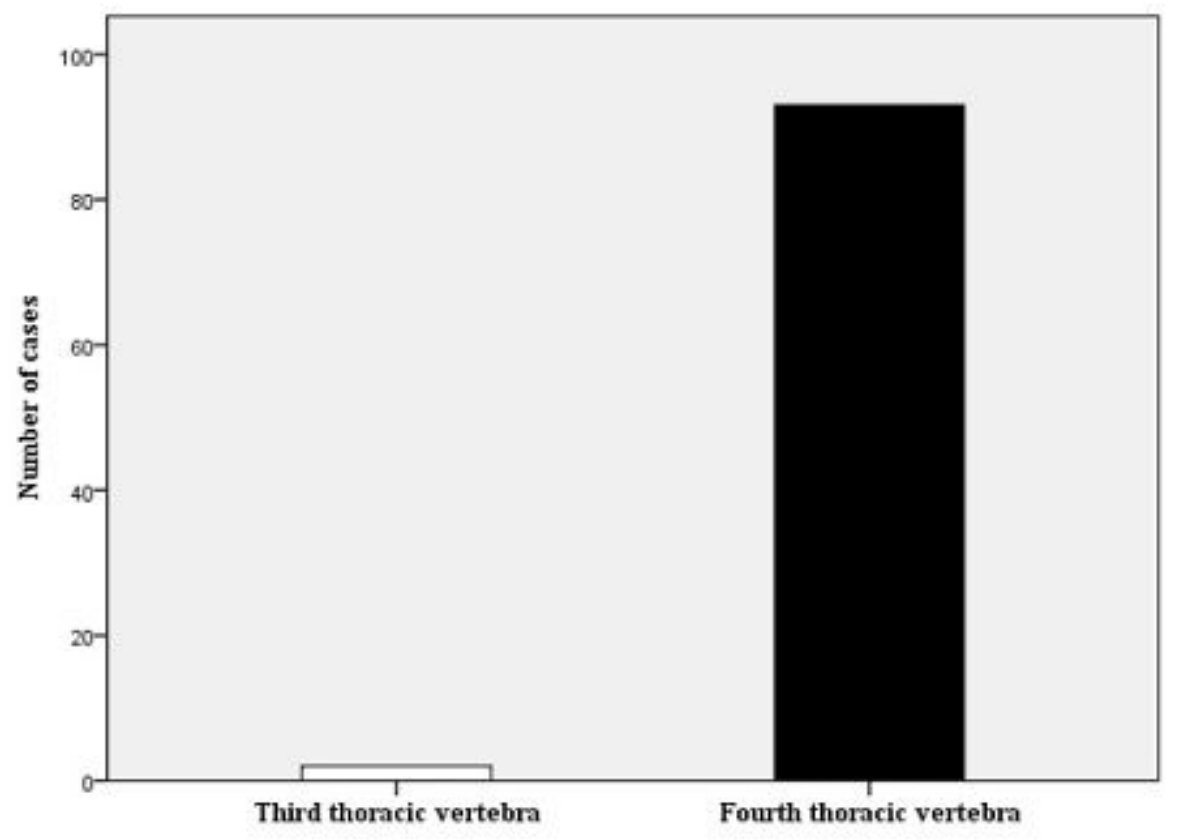

Figure 3

Positional relationship between the carina and thoracic vertebra in 95 infants

N.B.: the carina was located at the fourth thoracic vertebra more than in other locations, $P<0.05$

\section{Supplementary Files}

This is a list of supplementary files associated with this preprint. Click to download.

- Highlights.docx 